\title{
Ductal Carcinoma in situ after Core Needle Biopsy: In Which Cases Is a Sentinel Node Biopsy Necessary?
}

\author{
Robbert J.H. van Leeuwen Birgitta Kortmann Herman Rijna \\ Department of Surgery, Spaarne Gasthuis, Haarlem, The Netherlands
}

\author{
Keywords \\ Ductal carcinoma in situ - Sentinel node biopsy · Invasive \\ breast cancer
}

\begin{abstract}
Introduction: In some hospitals it is still common practice to carry out a sentinel node biopsy (SNB) if ductal carcinoma in situ (DCIS) is determined in preoperative staging, although this is against international guidelines. The reason for this is because an infiltrative component can be demonstrated frequently in the final pathohistological examination. In this study, we wanted to investigate possible predictors for infiltrative growth, to select patients to do an SNB or to omit it. Material and Methods: All patients with DCIS in the core needle biopsy (CNB), who were treated with surgery including an SNB, were included in a prospective data registry. Patient characteristics were collected through physical examination, mammography and ultrasonography. All characteristics of the DCIS were noted. After surgery, the pathological results were collected. Results: From the 287 patients, 39 (13.6\%) had an infiltrative component in the definitive pathological examination despite only DCIS in preoperative CNB. In total, there were only 14 (4.9\%) positive SNBs, of which 11 patients had infiltrative growth in the breast tumor and 3 (1.2\% of patients with DCIS alone in the final pathology) did not. In addition, characteristics of the CNB, including microcalcifications and comedonecrosis, did not show a statistically significant higher risk for infiltration. Discussion: Considering the low rates of positive SNBs in our population, we think that an SNB should not be performed in advance when DCIS is diagnosed,
\end{abstract}

because if infiltrative growth is found in the final biopsy, an SNB could always be performed afterwards. Only if an SNB cannot be performed afterwards is an SNB indicated.

(c) 2019 S. Karger AG, Basel

\section{Introduction}

Ductal carcinoma in situ (DCIS) is increasingly diagnosed, in particular since the introduction of screening programs in multiple countries around the world [1-3]. Since the 1970s, an increase of around 500\% has been reported [4].

In general, DCIS is seen as a precursor for the development of an invasive ductal carcinoma $[2,5]$ and therefore is treated in the same way as breast cancer: by lumpectomy or mastectomy and sentinel lymph node biopsy (SNB). An SNB was advised because in 11-25\% of the cases where a core needle biopsy (CNB) diagnosed only a DCIS the final lumpectomy demonstrated an invasive component [6-11]. Multiple studies have confirmed this presumption and have concluded that an SNB should not be a standard procedure $[12,13]$.

Nevertheless, the number of positive SNB after DCIS is low, and an SNB has several possible complications including lymphedema (6.9\%), anaphylactic shocks from the used contrast fluid (blue dye or radiocolloids; $0.1 \%$ ), wound infections (1-2\%) and axillary seromas (6-7.1\%) $[14,15]$. Furthermore 6 months after the performance of an SNB, 8.6-12\% of patients reported axillary paresthesias, and $3.8 \%$ reported a decreased range of motion of the 
upper extremity. Therefore, it is possible that an SNB is overtreatment.

In the most recent guideline of the American Society of Clinical Oncology, it has been attempted to find certain conditions to determine whether an SNB has to be conducted or not. They state that in DCIS, which requires a mastectomy, lesions highly suspected for invasive tumor and lesions bigger than $5 \mathrm{~cm}$ deserve an SNB. However, the evidence for these circumstances is weak to moderate [16]. The same kind of study was conducted by Fraile et al. [17], who tried to refine the circumstances for conducting an SNB. In this study, only high-risk DCIS patients were included, and no statistically significant factors were demonstrated [17]. Recently 2 studies have drawn similar conclusions. Francis et al. [18] concluded that SNB should only be conducted with patients that have a high risk of an invasive disease, for example, those with high-grade lesions or lesions bigger than $4 \mathrm{~cm}$. According to another study, SNB should no longer be performed. The study states that it is unnecessary, because it can always be performed afterwards, if the final histopathology revealed invasive growth [19].

In this study, we researched the number of positive SNBs in patients with DCIS in the core biopsy and tried to define the exact circumstances for an SNB to be positive postoperatively. If these circumstances can be determined, it would be possible to save patients from overtreatment and the unwanted side effects and complications of an SNB.

\section{Methods}

In this study, we included patients who were treated in the period between January 1, 2009, and June 1, 2016, using a prospective data registry. Patients were included when an isolated DCIS was present in their CNB and a lumpectomy or mastectomy with SNB was conducted subsequently. The results of the DCIS were graded in low, intermediate or high risk, according to the protocol of Lester et al. [20]. Patients who already had a history of breast cancer, had a bilateral lesion or patients who had other malignancies were excluded from the data set. Data reported in our study were taken from physical examination (i.e., the size of the possible palpable tumor, the presence or absence of lymphadenopathy), mammography and ultrasonography (the presence of a solid mass, its size, lymphadenopathy and the presence of microcalcifications).

Histological samples were taken with a 14-gauge core needle. When microcalcifications were present, this was conducted with a stereotactic vacuum biopsy; otherwise it was done with the support of ultrasound. The size of the tumor, the grade of DCIS and radical resection afterwards were added to the database. A radical resection was a resection with a margin of $>2 \mathrm{~mm}$ and was noted as an "R0 resection." Subsequently, the SNB was determined to be either positive or negative. If it were to be positive, the grade of the SNB (micrometastasis, macrometastasis or an N1 metastasis) was imported into the data set.

In the end, all results were imported in an SPSS data file and analyzed. We calculated means and tried to prove statistical sig- nificance with a $\chi^{2}$ test for 2 nominal variables and the $t$ test for normally divided variables. Multivariable analyses were performed with a one-way MANOVA test. A difference was assumed to be statistically significant if the $p$ value was smaller than 0.05 .

The study was approved by the hospital's ethicals board. An informed consent was not asked, because of the anonymity of the study. Names of the patients were not available, for none of the researchers.

\section{Results}

Five thousand three hundred and seventy-seven patients from the period between January 2009 and June 2016, who had the code breast malignancy, were collected from the digital patient system EPIC ${ }^{\circledR}$. Two hundred eighty-seven patients had a DCIS in CNB and were treated with a lumpectomy or mastectomy with an SNB. Of these 287 women who were included (Table 1), the mean age was 59.7 years with an SD of 9.3 years. Forty patients had a palpable tumor, with a mean size of $2.2 \mathrm{~cm}$ (SD 1.7), 216 did not have a palpable tumor and of 31 patients the information was not reported. On mammography, 74 patients had a solid tumor with a mean size of $2.6 \mathrm{~cm}$ (SD 2.0), 213 had no solid mass on radiographs. On ultrasonography, 58 patients had a solid mass, 194 did not and in 35 cases no ultrasonography was made. The mean size of a mass on ultrasonography was $1.7 \mathrm{~cm}$ (SD 1.8).

In preoperative histological biopsy, 279 patients had microcalcifications in the $\mathrm{CNB}, 8$ did not. Comedonecrosis was found in 110 biopsies and was not found in 162 . Fifteen biopsies were not documented. Twenty-eight patients had a grade 1 DCIS in the biopsy, 158 had a grade 2 and 99 patients had a grade 3 DCIS. Two files were not reported.

Two hundred and three patients underwent breastconserving surgery, 84 a mastectomy. The postoperative histological results showed 39 cases positive for carcinoma (13.9\%). In 248 cases, only DCIS was found. Of the infiltrative carcinomas, the mean size of the infiltrative component was $0.7 \mathrm{~cm}$ (SD 0.7). In the cases of an infiltrative component, the mean size of the total tumor was $3.0 \mathrm{~cm}$ (SD 0.7). In the noninfiltrative tumors the mean size was $2.4 \mathrm{~cm}$ (SD 2.8). A bigger size of the preoperatively diagnosed tumor did not give a higher risk for infiltrative growth $(p=0.143)$. In $48(16.7 \%)$, DCIS tissue was found within the resection margin of $2 \mathrm{~mm}$, and so this was called an R1 resection. In 240 cases (83.3\%) it was an R0.

Finally, the SNB was positive in $4.9 \%$ of the cases $(n=$ 14), of which in 8 cases a micrometastasis was found, and in 6 cases a macrometastasis. Nine times a BCS was conducted, 5 times a mastectomy. In 11 of the 14 cases, the lumpectomy or mastectomy showed an infiltrative component, in $3(1 \%)$ cases a positive SNB was found despite 
Table 1. Patient data

\begin{tabular}{|c|c|c|c|c|c|c|c|}
\hline & DCIS & Carcinoma & $p$ value & SNP- & $\mathrm{SNP}+$ & Missing & $p$ value \\
\hline \multicolumn{8}{|l|}{ Patient characteristics } \\
\hline Mean age (SD), years & $60.0(10.1)$ & $58.2(10.1)$ & 0.730 & $59.8(9.4)$ & $58.1(9.4)$ & 0 & 0.643 \\
\hline Women $<55$ years & 86 & 20 & & 98 & 8 & 0 & \\
\hline Women $>55$ years & 162 & 19 & 0.048 & 174 & 6 & 0 & 0.111 \\
\hline \multicolumn{8}{|l|}{ Physical examination } \\
\hline \multicolumn{8}{|l|}{ Palpability } \\
\hline Yes & 34 & 6 & & 39 & 1 & 31 & \\
\hline No & 189 & 27 & 0.665 & 205 & 11 & 31 & 0.476 \\
\hline \multicolumn{8}{|l|}{$\mathrm{X}$-ray solid mass } \\
\hline Yes & 65 & 9 & & 76 & 3 & 0 & \\
\hline No & 183 & 30 & 0.865 & 198 & 11 & 0 & 0.879 \\
\hline \multicolumn{8}{|l|}{ Echography solid mass } \\
\hline Yes & 47 & 11 & & 55 & 3 & 35 & \\
\hline No & 169 & 25 & 0.252 & 183 & 10 & 35 & 0.998 \\
\hline \multicolumn{8}{|l|}{ Core needle biopsy } \\
\hline \multicolumn{8}{|l|}{ Microcalcifications } \\
\hline Yes & 241 & 38 & & 265 & 14 & 1 & \\
\hline No & 7 & 1 & 0.963 & 7 & 0 & 1 & 0.543 \\
\hline \multicolumn{8}{|l|}{ Comedonecrosis } \\
\hline Yes & 93 & 17 & & 103 & 7 & 15 & \\
\hline No & 143 & 19 & 0.384 & 156 & 6 & 15 & 0.319 \\
\hline \multicolumn{8}{|l|}{ Grade of DCIS } \\
\hline 1 & 23 & 5 & & 26 & 2 & 2 & \\
\hline 2 & 140 & 18 & & 152 & 6 & 2 & \\
\hline 3 & 83 & 16 & 0.457 & 93 & 6 & 2 & 0.617 \\
\hline \multicolumn{8}{|l|}{ Type of surgery } \\
\hline Breast sparing & 175 & 28 & & 194 & 9 & 0 & \\
\hline Mastectomy & 73 & 11 & 0.863 & 79 & 5 & 0 & 0.593 \\
\hline Radical resection (R0) & 209 & 31 & & 227 & 13 & 0 & \\
\hline Nonradical resection (R1) & 39 & 8 & 0.459 & 46 & 1 & 0 & 0.336 \\
\hline
\end{tabular}

DCIS, ductal carcinoma in situ; SNP, single nucleotide polymorphism.

the fact that in the specimen an infiltrative component could not be found.

All preoperative variables were compared with the postoperative histological diagnosis. The only statistically significant difference we found was that women younger than 55 years had a greater risk of an infiltrative component $(p=0.048)$. All other variables showed no statistically significant differences and are not precursors for infiltrative components, even if variables were combined. Also, a multivariate analysis with the one-way MANOVA test did not show any differences for all investigated variables.

\section{Discussion}

In the final pathohistological examination, we found an infiltrative component in $13.9 \%$ of the cases, and a positive SNB in $4.9 \%$ of the cases. These numbers are similar to other studies, from 0.0 to $42.3 \%$ for infiltrative growth
[21], and a positive SNB in $5.5-10.7 \%$ of the cases $[18,19$, 22].

In our study we wanted to select a group of patients with a preoperative diagnosis of DCIS, who have a higher or lower risk of infiltrative growth and so have a higher or lower risk of a positive sentinel node. According to our data, such precursors are not available. Even in the literature, a clear variable that predicts this infiltrative growth has not been found until now. In another Dutch study by van Roozendaal et al. [19], the size of the needle biopsy whereby the $\mathrm{CNB}$ was performed showed a significantly different chance of finding infiltration. Another study shows a significantly higher risk of infiltration in the surgical specimen if sclerosing adenosis is found in the biopsy, pleiomorphic calcifications are found on the mammogram or a "suspect" mass is found on echography or if a mass is bigger than $2 \mathrm{~cm}$ [23]. However, these variables only predict a higher risk and they do not guarantee infiltration. 
Another predictor of infiltrative growth is the size of the lesion which was preoperatively measured. In our study, this difference was not significant. In guidelines, it is suggested that lesions $>2.5 \mathrm{~cm}$ have a higher risk for infiltrative growth and so have a higher risk for a positive SNB [24]. Though, not in all studies this significance is found [19].

Also in this study, comedonecrosis tends to give a higher risk for invasive growth [23]. In our study, we did not find this trend. Finally, an age younger than 55 years was reported as an independent factor for finding infiltration [7], which is similar to the results we found in our data. However, there was no correlation with a significant higher risk to have a positive SNB. Because of this unpredictability of infiltration and the low rates of positive SNB, we think that an SNB has to be conducted only for the cases where it cannot be conducted afterwards, like a mastectomy or a lateral upper quadrantectomy. After all, when you solely find DCIS, there is only a low risk of $4 \%$ that the SNB is positive.

By doing so, patients will be saved from SNBs and thereby from common complications like wound infections, axillary seromas and paresthesias [14, 15]. Except for the possible complications, also a rate of false-negative SNBs around 5\% was found in the literature [25]. Finally, the quality of life was found to be significantly lower for patients who had undergone an SNB in contrast to patients who had not [26].

Furthermore there is a discussion whether an SNB is still necessary when an infiltrative component is found. Recent results show that watchful waiting, in comparison to performing an axillary lymph node dissection, shows no significant differences in regional recurrence rates after a follow-up of 6.3 years [26]. And even when, during follow-up, lymphatic metastases develop, an additional axillary lymph node dissection at a later time does not show any differences in overall survival or disease-free interval $[27,28]$.

Except for the existing parameters, there may be some new parameters which can help to determine the risk for invasive growth. First, the histopathological status can be determined and can divide the types of DCIS in molecular phenotypes: Luminal A (ER/PR+, Her2neu-), Luminal B (triple positive), Her2neu positive (ER/PR-, Her2neu+) and triple negative. These types of DCIS were researched by Williams et al. [29], who showed that the Luminal A type has a much lower recurrence and invasive recurrence rate compared to the Her2neu positive type. Another study showed a higher recurrence rate when a Ki-67 hormone receptor was found [30].

In addition to the hormonal status, special applications of magnetic resonance imaging can help in differentiating DCIS from infiltrating breast cancer, which was shown in a study by Ding et al. [31]. In this study, diffused weighted imaging was used, which is a way of imaging in order to determine the degree of swelling of cells. Diffused weighted imaging can show this difference, but only in the Asian population and not in the Caucasian [31]. In another study it was shown that using the magnetic resonance imaging scan this way may contribute to the differentiation between invasive and in situ breast carcinoma [32].

One of the limitations of this study is clearly its retrospective design. Although this design leads to the high number of study subjects, retrospective cohort studies are known for their selection bias of the studied population. In our study, we tried to prevent this bias by clear in- and exclusion criteria.

In conclusion, until now there has been no clear way to definitively predict whether a tumor is a DCIS or an invasive tumor. For that reason, we have to deal with the evidence that is available at the moment. We advise, taking into account our data and the literary study, to do a SNB only when this will not be possible anymore in a later stadium, which means that when a mastectomy or a lateral upper quadrantectomy has been performed or when a patient is not able to survive 2 separate operations. In general lumpectomies, an SNB should always be possible after the definitive histology of the lumpectomy. If implemented, this protocol will cause no undertreatment of DCIS lesions, and patients do not need to undergo unnecessary or invasive treatment for a benign lesion.

\section{Acknowledgments}

We thank pathologist Dr. K. van Groningen for his contribution to this study. He helped us collecting all the pathological data.

\section{Statement of Ethics}

This study was approved by the hospital's internal ethics board. Informed consent was not necessary because of the anonymity of the usage of patient data. The study was conducted fully anonymously, none of the authors had insight into the patients' private data.

\section{Disclosure Statement}

The authors have no conflicts of interest to declare.

\section{Author Contributions}

R.J.H.v.L. was the main author of this study and was supervised by Dr. B. Kortmann and Dr. H. Rijna. Both supervisors helped by collecting the data and made corrections to the final text. According to the ICMJE Guidelines, they can be seen as co-authors. 


\section{References}

1 Brinton LA, Sherman ME, Carreon JD, Anderson WF. Recent trends in breast cancer among younger women in the United States. J Natl Cancer Inst. 2008 Nov;100(22):1643-8.

2 Virnig BA, Tuttle TM, Shamliyan T, Kane RL. Ductal carcinoma in situ of the breast: a systematic review of incidence, treatment, and outcomes. J Natl Cancer Inst. 2010 Feb; 102(3):170-8

3 Ward EM, DeSantis CE, Lin CC, Kramer JL, Jemal A, Kohler B, et al. Cancer statistics: breast cancer in situ. CA Cancer J Clin. 2015 Nov-Dec;65(6):481-95.

4 Ernster VL, Barclay J, Kerlikowske K, Grady $\mathrm{D}$, Henderson C. Incidence of and treatment for ductal carcinoma in situ of the breast. JAMA. 1996 Mar;275(12):913-8.

5 Sagara Y, Mallory MA, Wong S, Aydogan F, DeSantis S, Barry WT, et al. Survival Benefit of Breast Surgery for Low-Grade Ductal Carcinoma In Situ: A Population-Based Cohort Study. JAMA Surg. 2015 Aug;150(8):739-45.

6 Dominguez FJ, Golshan M, Black DM, Hughes KS, Gadd MA, Christian R, et al. Sentinel node biopsy is important in mastectomy for ductal carcinoma in situ. Ann Surg Oncol. 2008 Jan;15(1):268-73.

7 Yen TW, Hunt KK, Ross MI, Mirza NQ, Babiera GV, Meric-Bernstam F, et al. Predictors of invasive breast cancer in patients with an initial diagnosis of ductal carcinoma in situ: a guide to selective use of sentinel lymph node biopsy in management of ductal carcinoma in situ. J Am Coll Surg. 2005 Apr;200(4):516-26.

8 Cox CE, Nguyen K, Gray RJ, Salud C, Ku NN, Dupont $\mathrm{E}$, et al. Importance of lymphatic mapping in ductal carcinoma in situ (DCIS): why map DCIS? Am Surg. 2001 Jun;67(6): 513-9.

9 Brennan ME, Turner RM, Ciatto S, Marinovich ML, French JR, Macaskill P, et al. Ductal carcinoma in situ at core-needle biopsy: meta-analysis of underestimation and predictors of invasive breast cancer. Radiology. $2011 \mathrm{Jul}$; 260(1):119-28.

10 Kettritz U, Rotter K, Schreer I, Murauer M, Schulz-Wendtland R, Peter D, et al. Stereotactic vacuum-assisted breast biopsy in 2874 patients: a multicenter study. Cancer. 2004 Jan; 100(2):245-51.

11 Houssami N, Ciatto S, Bilous M, Vezzosi V, Bianchi S. Borderline breast core needle histology: predictive values for malignancy in lesions of uncertain malignant potential (B3). Br J Cancer. 2007 Apr;96(8):1253-7.

12 Intra $\mathrm{M}$, Rotmensz $\mathrm{N}$, Veronesi P, Colleoni M, Iodice S, Paganelli G, et al. Sentinel node biopsy is not a standard procedure in ductal carcinoma in situ of the breast: the experience of the European institute of oncology on 854 patients in 10 years. Ann Surg. 2008 Feb 247(2):315-9.
13 Zavagno G, Carcoforo P, Marconato R, Franchini Z, Scalco G, Burelli P, et al. Role of axillary sentinel lymph node biopsy in patients with pure ductal carcinoma in situ of the breast. BMC Cancer. 2005 Mar;5(1):28.

14 Wilke LG, McCall LM, Posther KE, Whitworth PW, Reintgen DS, Leitch AM, et al. Surgical complications associated with sentinel lymph node biopsy: results from a prospective international cooperative group trial. Ann Surg Oncol. 2006 Apr;13(4):491-500.

15 Lucci A, McCall LM, Beitsch PD, Whitworth PW, Reintgen DS, Blumencranz PW, et al.; American College of Surgeons Oncology Group. Surgical complications associated with sentinel lymph node dissection (SLND) plus axillary lymph node dissection compared with SLND alone in the American College of Surgeons Oncology Group Trial Z0011. J Clin Oncol. 2007 Aug;25(24):3657-63.

16 Lyman GH, Temin S, Edge SB, Newman LA, Turner RR, Weaver DL, et al.; American Society of Clinical Oncology Clinical Practice. Sentinel lymph node biopsy for patients with early-stage breast cancer: american Society of Clinical Oncology clinical practice guideline update. J Clin Oncol. 2014 May;32(13):136583

17 Fraile M, Gubern JM, Rull M, Julián FJ, Serra C, Llatjós M, et al. Is it possible to refine the indication for sentinel node biopsy in highrisk ductal carcinoma in situ? Nucl Med Commun. 2006 Oct;27(10):785-9.

18 Francis AM, Haugen CE, Grimes LM, Crow JR, Yi M, Mittendorf EA, et al. Is Sentinel Lymph Node Dissection Warranted for Patients with a Diagnosis of Ductal Carcinoma In Situ? Ann Surg Oncol. 2015 Dec;22(13): 4270-9.

19 van Roozendaal LM, Goorts B, Klinkert M, Keymeulen KB, De Vries B, Strobbe LJ, et al. Sentinel lymph node biopsy can be omitted in DCIS patients treated with breast conserving therapy. Breast Cancer Res Treat. 2016 Apr; 156(3):517-25.

20 Lester SC, Bose S, Chen YY, Connolly JL, de Baca ME, Fitzgibbons PL, et al.; Members of the Cancer Committee, College of American Pathologists. Protocol for the examination of specimens from patients with ductal carcinoma in situ of the breast. Arch Pathol Lab Med. 2009 Jan;133(1):15-25.

21 El Hage Chehade H, Headon H, Wazir U, Abtar H, Kasem A, Mokbel K. Is sentinel lymph node biopsy indicated in patients with a diagnosis of ductal carcinoma in situ? A systematic literature review and meta-analysis. Am J Surg. 2017 Jan;213(1):171-80.

22 Prendeville S, Ryan C, Feeley L, O’Connell F, Browne TJ, O'Sullivan MJ, et al. Sentinel lymph node biopsy is not warranted following a core needle biopsy diagnosis of ductal carcinoma in situ (DCIS) of the breast. Breast. 2015 Jun;24(3):197-200
23 Kondo T, Hayashi N, Ohde S, Suzuki K, Yoshida A, Yagata $\mathrm{H}$, et al. A model to predict upstaging to invasive carcinoma in patients preoperatively diagnosed with ductal carcinoma in situ of the breast. J Surg Oncol. 2015 Oct;112(5):476-80.

24 Kaufman SA, Harris EE, Bailey L, Chadha M, Dutton SC, Freedman GM, et al. ACR appropriateness criteria ${ }^{\circledR}$ ductal carcinoma in situ. Oncology (Williston Park). 2015 Jun;29(6): 446-58, 460-1.

25 McMasters KM, Tuttle TM, Carlson DJ, Brown CM, Noyes RD, Glaser RL, et al. Sentinel lymph node biopsy for breast cancer: a suitable alternative to routine axillary dissection in multi-institutional practice when optimal technique is used. J Clin Oncol. 2000 Jul; 18(13):2560-6

26 Fallowfield LJ. Evolution of breast cancer treatments: current options and quality-oflife considerations. Eur J Oncol Nurs. 2004;8 Suppl 2:S75-82.

27 Giuliano AE, McCall L, Beitsch P, Whitworth PW, Blumencranz P, Leitch AM, et al. Locoregional recurrence after sentinel lymph node dissection with or without axillary dissection in patients with sentinel lymph node metastases: the American College of Surgeons Oncology Group Z0011 randomized trial. Ann Surg. 2010 Sep;252(3):426-32.

28 Galimberti V, Monti S, Mastropasqua MG. DCIS and LCIS are confusing and outdated terms. They should be abandoned in favor of ductal intraepithelial neoplasia (DIN) and lobular intraepithelial neoplasia (LIN). Breast. 2013 Aug;22(4):431-5.

29 Williams KE, Barnes NL, Cramer A, Johnson $\mathrm{R}$, Cheema K, Morris J, et al. Molecular phenotypes of DCIS predict overall and invasive recurrence. Ann Oncol. 2015 May;26(5): 1019-25.

30 Poulakaki N, Makris GM, Battista MJ, Böhm D, Petraki K, Bafaloukos D, et al. Hormonal receptor status, Ki-67 and HER2 expression: prognostic value in the recurrence of ductal carcinoma in situ of the breast? Breast. 2016 Feb;25:57-61.

31 Ding JR, Wang DN, Pan JL. Apparent diffusion coefficient value of diffusion-weighted imaging for differential diagnosis of ductal carcinoma in situ and infiltrating ductal carcinoma. J Cancer Res Ther. 2016 Apr-Jun; 12(2):744-50.

32 Nadrljanski M, Maksimović R, PlešinacKarapandžić V, Nikitović $M$, MarkovićVasiljković B, Milošević Z. Positive enhancement integral values in dynamic contrast enhanced magnetic resonance imaging of breast carcinoma: ductal carcinoma in situ vs. invasive ductal carcinoma. Eur J Radiol. 2014 Aug; 83(8):1363-7. 